\title{
Facoemulsificação: resultados e complicações nos primeiros 100 olhos
}

\author{
Phacoemulsification: outcomes and complications in the first 100 eyes
}

\author{
Maria Emília Xavier dos Santos Araújo ${ }^{(1)}$ \\ André Chang' Chou ${ }^{(2)}$ \\ Clebert Reinaldo da Silva ${ }^{(3)}$ \\ Leonardo Bruno de Oliveira ${ }^{(3)}$ \\ Isaac Neustein ${ }^{(4)}$
}

\begin{tabular}{|l|}
\hline \multicolumn{1}{|c|}{ RESUMO } \\
\hline Objetivo: Analisar o resultado visual e as complicações dos 100 \\
primeiros olhos submetidos à facoemulsificação, realizados no Hos- \\
pital do Servidor Público Estadual de São Paulo (HSPE), por cirur- \\
giões iniciantes nesta técnica. \\
Pacientes eMétodo: As 100 primeiras cirurgias de facoemulsificação \\
realizadas por 05 cirurgiões, em pacientes do ambulatório de Cata- \\
rata do HSPE, foram analisadas. A técnica utilizada foi incisão \\
tunelizada, capsulorrexis curvilínea contínua anterior, facoemulsifi- \\
cação do núcleo, usando um aparelho de bomba peristáltica, e \\
implante de lente intra-ocular, sob anestesia com bloqueio peribulbar. \\
Resultados: As complicações ocorreram em $15,2 \%$ dos olhos, \\
sendo rotura de cápsula posterior sem perda vítrea em $8,7 \%$, perda \\
vítrea em 5,4\% e luxação de núcleo no vítreo em 1 olho. Em $96,7 \%$ dos \\
olhos foram implantados LIO de câmara posterior, sendo $70,8 \%$ no \\
saco capsular, $28 \%$ no sulco, $1,2 \%$ fixação escleral superior. A \\
acuidade visual final foi maior que $20 / 40$ em $89 \%$ dos olhos e nenhum \\
evoluiu para ceratopatia bolhosa ou descolamento de retina. \\
Conclusão: A incidência de complicações foi comparável a da \\
literatura, sugerindo que o aprendizado pode ser iniciado no período \\
da residência médica. \\
Palavras-chave: Facoemulsificação; Curva de aprendizado; Complicação.
\end{tabular}

\section{INTRODUÇ̃̃̃O}

O estágio atual da cirurgia de catarata, com a facoemulsificação, oferece ao paciente um excelente resultado visual e recuperação precoce. Nos últimos anos, nos Estados Unidos (EUA), tem se tornado o procedimento de escolha para extração da catarata, com crescente aumento do número de cirurgiões usando esta técnica ${ }^{1}$. A curva de aprendizado, na transição da técnica da cirurgia extracapsular para a facoemulsificação, pode ser iniciada na residência médica, com o preparo adequado do cirurgião que deseja atingir experiência e domínio desta técnica.

O objetivo deste estudo é avaliar os resultados e complicações das 100 primeiras cirurgias de catarata, por facoemulsificação, realizadas no Hospital do Servidor Público Estadual de S. Paulo.

\section{MATERIAL E MÉTODOS}

Foram analisados os 100 primeiros olhos submetidos à cirurgia de 
facoemulsificação com implante de lente intraocular, efetuados por cirurgiões que começaram a realizar esta técnica (iniciantes), com, pelo menos, um ano de experiência em extração extracapsular (EEC) tunelizada ${ }^{2}$. A idade dos pacientes variou entre 38 a 84 anos, com média de 61,4 anos, sendo 68 do sexo feminino e 32 , masculino. A acuidade visual pré-operatória variou de conta dedos a 20/50, com a melhor correção (Gráfico I). O treinamento dos cirurgiões em capsulorrexis e facoemulsificação constou de cursos teóricos com vídeos e aulas ministradas por cirurgiões experientes, e práticas em laboratório experimental ("wet lab"). A capsulorrexis também foi treinada nas cirurgias de EEC previamente à mudança da técnica. Pacientes com apenas um olho, com prognóstico visual pobre, doenças prévias do segmento anterior como sinéquias, cristalino subluxado, opacidades significantes de córnea, bolhas filtrantes e pouca dilatação pupilar, não foram incluídos no estudo.

O método padronizado consistiu em anestesia por bloqueio peribulbar, usando xilocaína a $2 \%$ e marcaína a $0,5 \%$, retopexia superior com seda 4.0 , peritomia de base fórnix, túnel escleral superior a $2 \mathrm{~mm}$ do limbo, com incisão reta de 6,5 a 7,0mm de acordo com o diâmetro da lente a ser implantada, paracentese à esquerda da incisão para a entrada do segundo instrumento, abertura inicial de $3,2 \mathrm{~mm}$, capsulorrexis curvilínea contínua anterior com agulha de insulina e/ou pinça, com a proteção de viscoelástico (hialuronato de sódio), hidrodissecção do núcleo com cânula e solução de ringer, facoemulsificação do núcleo com a técnica "flip and chip" ${ }^{3}$ ou "divide and conquer" 4 , usando um aparelho de facoemulsificação, bomba peristáltica, que não possui mecanismo de controle de manutenção da câmara anterior (modelo Harmony Special Phaco, versão 2.11 da Dutch Ophthalmic Research Center - D.O.R.C.) ${ }^{\mathrm{R}}$. Os parâmetros utilizados na fase de esculpir o núcleo foram: poder de ultra-som de 50\%, linear, limite de vácuo igual a $17 \mathrm{mmHg}$ e fluxo de aspiração de 20

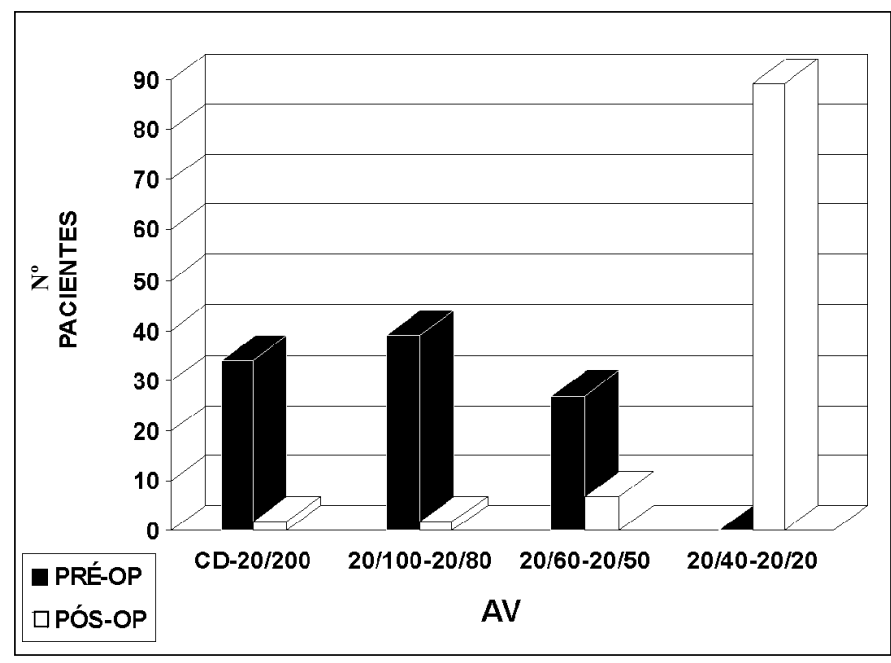

Gráfico I - Acuidade visual dos pacientes submetidos à facoemulsificação $\mathrm{ml} /$ minuto. Na aspiração de fragmentos do núcleo o limite de vácuo foi $167 \mathrm{mmHg}$, mantendo-se inalterados o poder de ultra-som e o fluxo. A aspiração do córtex foi realizada com a caneta de irrigação-aspiração ou cânula de Simcoe, ampliação da incisão e implante da lente intraocular de polimetilmetacrilato - PMMA (SAPHIR 652 - OPSIA e STORZ BVR$170 \mathrm{~L})^{\mathrm{R}}$, com diâmetro de zona, óptica entre 6,5 a 7,0 mm; sutura de 1 a 3 pontos separados, radiais, com mononylon 10.0. Utilizou-se ringer lactato como solução de irrigação em todos os casos, e, em alguns, adrenalina ( $4 \mathrm{mg} / \mathrm{ml}$ diluída em $250 \mathrm{ml}$ da solução de irrigação) para ampliar a midríase. Ao final da cirurgia foi aplicada injeção de gentamicina e dexametasona subconjuntival. Realizou-se vitrectomia anterior com vitreófago e implante de lente intra-ocular (LIO) no sulco capsular, nos olhos com perda de humor vítreo.

\section{RESULTADOS}

As cirurgias foram realizadas entre agosto de 1997 e janeiro de 1998. Dos 100 olhos operados, 8 cirurgias foram convertidas para a técnica EEC, sendo $6 \mathrm{em}$ virtude de capsulorrexis descontínuas, 1 por dificuldade na facoemulsificação e 1 por rotura de cápsula posterior.

As complicações ocorreram em 14 olhos (15,2\%), com rotura de cápsula sem perda vítrea em $8(8,7 \%)$, com perda vítrea em $5(5,4 \%)$, sendo em 9 olhos no momento da aspiração do córtex e 4 na facoemulsificação do núcleo, e em um, núcleo no vítreo (Gráfico II); este último foi submetido à vitrectomia e implante de LIO, em segundo tempo, com bom resultado visual (AV 20/25cc). Em 96,7\% dos olhos foram implantados LIO de câmara posterior, sendo $70,8 \%$ no saco capsular, $28 \%$ no sulco e 1,2\% (1 olho), fixação escleral; em 2 olhos (2,2\%), LIO de câmara anterior; em 1 (1,1\%) não foi implantada porque estava programada cirurgia de retina posteriormente. Em alguns olhos com LIO no sulco, a opção foi do cirurgião, para não ampliar a capsulorrexis.

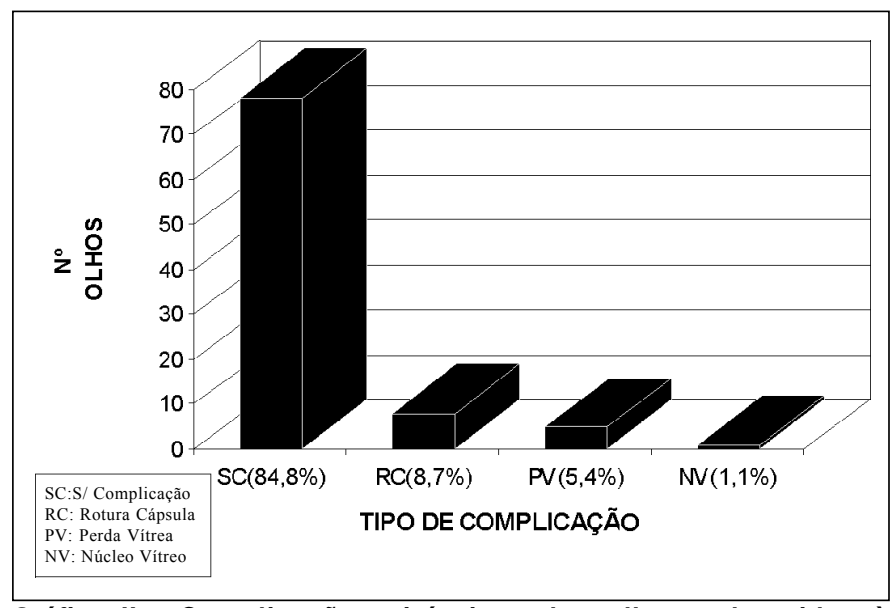

Gráfico II - Complicações cirúrgicas dos olhos submetidos à facoemulsificação 


\begin{tabular}{|c|c|c|c|c|}
\hline \multicolumn{5}{|c|}{$\begin{array}{l}\text { Tabela1. Resultado visual e \% de rotura de cápsula nos olhos } \\
\text { submetidos à facoemulsificação, em diferentes trabalhos } \\
\text { publicados. }\end{array}$} \\
\hline Autor & Ano & No Olhos & $\begin{array}{c}\text { Rotura } \\
\text { cápsula } \mathrm{N}^{\circ}(\%)\end{array}$ & $A V^{*}>20 / 40(\%)$ \\
\hline Dantas e col & 1995 & 50 & $12(18)$ & 84 \\
\hline Tarbet et al & 1995 & 300 & $17(5,6)$ & 90,6 \\
\hline Lambert e col & 1997 & 48 & $05(10)$ & 89,6 \\
\hline Robin et al & 1998 & 300 & $40(13,3)$ & $\mathrm{NR}^{* *}$ \\
\hline Prasad et al & 1998 & 102 & $06(5,6)$ & 90,5 \\
\hline Estudo atual & 1998 & 100 & $13(14,1)$ & 89 \\
\hline
\end{tabular}

O seguimento variou entre 3 e 8 meses de período pósoperatório, com refração prescrita, em média, no $2^{\circ}$ mês. A acuidade visual no período pós-operatório foi melhor que 20/40 em $89 \%$ dos olhos (Gráfico I). Os 11\% restante apresentavam degeneração macular relacionado à idade ( 8 olhos $)$, buraco macular (01) e retinopatia diabética (02). Nenhum olho evoluiu com ceratopatia bolhosa ou descolamento de retina no período de seguimento.

\section{DISCUSS ÃO}

Nos países desenvolvidos a facoemulsificação é, geralmente, ensinada durante a residência médica, com constante supervisão de cirurgiões experientes ${ }^{5}$. A crescente popularidade da técnica deve-se a diversos fatores como: a manutenção da arquitetura do segmento anterior; a redução no tamanho da incisão, levando à diminuição do astigmatismo induzido; um sistema fechado de cirurgia reduzindo a probabilidade de hemorragia coroidal expulsiva; a redução do tempo cirúrgico e a recuperação precoce das atividades pelo paciente.

Há uma tendência recente, para se iniciar o aprendizado da facoemulsificação sem o conhecimento anterior da EEC ${ }^{6}$. Entretanto, verificamos que a experiência prévia com a EEC garante ao cirurgião segurança para conversão em complicações, como foi verificado em $8 \%$ de nossos casos.

A curva de aprendizado tem estado associada a um maior número de complicações, mas estudos recentes ${ }^{5-10}$ (Tabela 1), têm mostrado uma queda neste índice. A rotura de cápsula posterior varia entre 4,8 a $11 \%$ e a perda vítrea entre 1,0 a $13,7 \%{ }^{7}$. Os parâmetros de funcionamento do equipamento utilizado (Harmony Special Phaco - D.O.R.C ) foram estabelecidos pelos cirurgiões e orientadores já que não havia similar no mercado. Apesar disso, os resultados foram semelhantes aos encontrados na literatura ${ }^{5-8,10}$ (Tabela 1 ), com rotura de cápsula sem perda vítrea em $8,7 \%$, e perda vítrea em 5,4\%. A acuidade visual final foi maior ou igual a 20/40 em $89 \%$ dos casos.

Facoemulsificação é procedimento seguro para ser ensinado na residência médica a cirurgiões com experiência na EEC, desde que todos os passos, como incisão tunelizada e capsulorrexis, sejam treinados previamente em cursos teóricos e práticos, e com uma criteriosa seleção de pacientes.

\section{SUMMARY}

Purpose: To analyze outcomes and incidence of complications in 100 eyes submitted to phacoemulsification performed by surgeons at the start of their career at the Hospital do Servidor Público Estadual de São Paulo.

Methods: The first 100 consecutive cataract operations performed by five surgeons, in patients of the HSPE, using phacoemulsification were evaluated. The surgical technique included a scleral tunnel, continuous curvilinear capsulorhexis, nucleus removal by phacoemulsification using a peristaltic pump and intraocular lens implantation, under peribulbar anesthesia.

Results: The incidence of complications was $15.2 \%$ : posterior capsule tear in $8.7 \%$, vitreous loss in $5.4 \%$ and one case of posterior dislocation of the nucleus. Posterior chamber IOL were implanted in $96.7 \%$ of the eyes, $70.8 \%$ of these being in the capsular bag, 28\% in the sulcus and $1.2 \%$ were fixed superiorly. The final visual acuity was 20/40 or better in $89 \%$ of the cases. None of them developed bullous keratopathy or retinal detachment.

Conclusion: The complication rate was comparable to that of the literature, suggesting that this technique may be learned during the residency program.

Keywords: Phacoemulsification; Learning curve; Complication.

\section{REFERÊNCIAS BIBLIOGRÁFICAS}

1. Leaming DV. Practice styles and preferences of ASCRS members-1996 survey. J Cataract Surg 1997; 17:527-35.

2. Emery J. Extracapsular cataract surgery. In: Steinert RF. Cataract Surgery: Technique, complications, \& management. W.B. Saunders company 1995;107-18.

3. Fine I. Chip and flip technique. In: Koch P, Davison J, eds. Textbook of advanced phacoemulsification techniques. Thorofare, NJ: Slack 1991:191-205.

4. Gimbel HV. Divide and conquer nucleofractis phacoemulsification: Development and variations. J cataract Refract Surg 1991;17:281-91.

5. Robin AL, Smith S, Natchiar G, Ramakrishnan R, Srinivasan, Raheem R, Hecht W. The initial complication rate of phacoemulsification in India. Investigative Ophthalmology \& Visual Science 1997;38:2331-7.

6. Tarbet KJ, Mamalis N, Theurer J, Jones BD, Olson RJ. Complications and results of phacoemulsification performed by residents. J Cataract Refract Surg, $1995 ; 21: 661-5$

7. Prasad S, FRCSEd. Phacoemulsification learning curve: Experience of two junior trainee ophthalmologists. J Cataract Refract Surg, 1998;24:73-7.

8. Lambert LC, Occhiutto ML, Paparelli CM, Kniggendorf S, Akaishi L, Mendonça $\mathrm{BD}$, Cvintal T. Resultados visuais e incidência de complicações em facoemulsificação com LIO por residentes. Rev Bras Oftal 1997;56(12):953-6.

9. Corey RP, Olson RJ. Surgical outcomes of cataract extractions performed by residents using phacoemulsification. J Cataract Refract Surg 1998;24:66-72.

10. Dantas PEC, Dantas MCN, Mandia Jr C, Waiswol M, Krasilchik G, Dias AKG. Facoemulsificação: A experiência da conversão - Análise dos 50 primeiros casos. Arq Bras Oftal 1995;58(5):421-4. 